\title{
Fixed Point Results for Generalized Fuzzy Contractive Mappings in Fuzzy Metric Spaces with Application in Integral Equations
}

\author{
Babak Mohammadi, ${ }^{1}$ Azhar Hussain, ${ }^{2}$ Vahid Parvaneh $\left(\mathbb{D},{ }^{3}\right.$ Naeem Saleem $\left(\mathbb{D},{ }^{4}\right.$ \\ and Rogheieh Jalal Shahkoohi ${ }^{5}$ \\ ${ }^{1}$ Department of Mathematics, Marand Branch, Islamic Azad University, Marand, Iran \\ ${ }^{2}$ Department of Mathematics, University of Sargodha, Sargodha 40100, Pakistan \\ ${ }^{3}$ Department of Mathematics, Gilan-E-Gharb Branch, Islamic Azad University, Gilan-E-Gharb, Iran \\ ${ }^{4}$ Department of Mathematics, University of Management and Technology, Lahore, Pakistan \\ ${ }^{5}$ Department of Mathematics, Aliabad Katoul Branch, Islamic Azad University, Aliabad Katoul, Iran
}

Correspondence should be addressed to Naeem Saleem; naeem.saleem2@gmail.com and Rogheieh Jalal Shahkoohi; rog.jalal@ gmail.com

Received 20 March 2021; Accepted 30 April 2021; Published 17 May 2021

Academic Editor: Ljubisa Kocinac

Copyright (c) 2021 Babak Mohammadi et al. This is an open access article distributed under the Creative Commons Attribution License, which permits unrestricted use, distribution, and reproduction in any medium, provided the original work is properly cited.

In this paper, we introduce generalized $\alpha$ - $\eta$-fuzzy contractive mappings and generalized $\beta$ - $\zeta$-fuzzy contractive mappings and prove existence of fixed point for such mappings. Our results generalize and improve the recent work of Gopal and Vetro (Iranian journal of fuzzy systems, 11 (2014), 95-107). Some equivalent conditions of our results are presented. Also, an example is given to support our new results.

\section{Introduction}

The notion of fuzzy sets was introduced initially by Zadeh [1] in 1965. Afterwards, Kramosil and Michalek [2] introduced the notion of fuzzy metric space (FMS). In 1994, George and Veeramani [3] modified the notion of FMS, introduced by Kramosil and Michalek [2], and presented a Hausdorff topology of a FMS. Recently, Samet et al. [4] introduced a new concept of $\alpha$-admissible and $\alpha$ - $\zeta$-contractive-type mappings which is described below.

Let $(\Pi, d)$ be a metric space. A mapping Q: $\Pi \longrightarrow \Pi$ is said to be an $\alpha-\zeta$-contractive mapping if there exist two functions $\alpha: \Pi^{2} \longrightarrow[0,+\infty)$ and $\zeta:[0,+\infty) \longrightarrow[0,+\infty)$ so that $\zeta$ is nondecreasing and $\sum_{n=1}^{\infty} \zeta^{n}(t)<\infty$, for all $t>0$, such that

$$
\alpha(\iota, \kappa) d(\mathbb{Q} l, \mathbb{Q} \kappa) \leq \zeta(d(\iota, \kappa))
$$

for all $\iota, \kappa \in \Pi$. They established and proved some fixed point theorems for such mappings in complete metric spaces. Very recently, motivated by Samet et al. [4], the concept of $\alpha$-admissible and $\alpha-\eta$-fuzzy contractive mappings and $\beta$ - $\zeta$-fuzzy contractive mappings in a FMS was discussed by Gopal and Vetro [5]. Gopal and Vetro introduced the concept of $\alpha-\eta$-fuzzy contractive mappings which is described below.

Let $(\Pi, \mathscr{F}, *)$ be a FMS. A mapping $Q$ : $\Pi \longrightarrow \Pi$ is called an $\alpha-\eta$-fuzzy contractive mapping if there exist two functions $\quad \alpha: \Pi^{2} \times(0,+\infty) \longrightarrow[0,+\infty)$ and $\eta:[0,+\infty) \longrightarrow[0,+\infty)$ such that $\eta$ is right continuous and $\eta(t)<t$, for all $t>0$, and

$$
\alpha(\iota, \kappa, t)\left(\frac{1}{\mathscr{F}(\mathbb{Q} \iota, Q \mathcal{Q} \kappa, t)}-1\right) \leq \eta\left(\frac{1}{\mathscr{F}(\iota, \kappa, t)}-1\right),
$$

for all $\iota, \kappa \in \Pi$ and for all $t>0$. 
In [6], the authors studied the existence of common $\alpha$-fuzzy fixed points for fuzzy mappings via F-contractions on a metric space. They obtained some common fixed points of fuzzy (multivalued) mappings satisfying an F-contraction associated with the Hausdorff metric.

In this paper, we introduce generalized $\alpha$ - $\eta$-fuzzy contractive and generalized $\beta$ - $\zeta$-fuzzy contractive mappings, which are motivated by Gopal and Vetro [5] and show that the fixed point exists for the presented mappings. Our results generalize and improve the results of Gopal and Vetro [5]. To show the usefulness of our results, some equivalent conditions are presented.

\section{Preliminaries}

In this section, we recall some definitions and notions which will be used in this paper.

Definition 1 (see [7]). A binary operation $*:[0,1]^{2} \longrightarrow[0,1]$ is a continuous t-norm if it satisfies the following conditions:

$(Q 1) *$ is associative and commutative

(Q2) $*$ is continuous

(Q3) $a * 1=a$ for all $a \in[0,1]$

(Q4) $a * b \leq c * d$ whenever $a \leq c$ and $b \leq d$ for all $a, b, c, d \in[0,1]$

Three examples of a continuous t-norm are Lukasievicz $\mathrm{t}$-norm, that is, $a *{ }_{L} b=\max \{a+b-1,0\}$, product t-norm, that is, $a *_{p} b=a b$, and minimum t-norm, that is, $a *{ }_{M} b=\min \{a, b\}$. The concept of FMS which was defined by George and Veeramani [3] which is as follows.

Definition 2 (see [3]). Let $\Pi$ be an arbitrary nonempty set, * be a continuous t-norm, and $\mathscr{F}$ be a fuzzy set on $\Pi^{2} \times(0, \infty)$. The triple $(\Pi, \mathscr{F}, *)$ is called a FMS if, for each $\iota, \kappa, z \in \Pi$ and for all $t, s>0$,

$$
\begin{aligned}
& (\mathscr{F} 1) \mathscr{F}(l, \kappa, t)>0 \\
& (\mathscr{F} 2) \mathscr{F}(l, \kappa, t)=1 \text { if and only if } \iota=\kappa \\
& (\mathscr{F} 3) \mathscr{F}(l, \kappa, t)=\mathscr{F}(\kappa, l, t) \\
& (\mathscr{F} 4) \mathscr{F}(l, z, t+s) \geq \mathscr{F}(l, \kappa, t) * \mathscr{F}(\kappa, z, s) \\
& (\mathscr{F} 5) \mathscr{F}(l, \kappa, .):(0, \infty) \longrightarrow[0,1] \text { is continuous }
\end{aligned}
$$

If property $(\mathscr{F} 4)$ be replaced by $\mathscr{F}(\iota, z, \max \{t, s\}) \geq \mathscr{F}(\iota, \kappa, t) * \mathscr{F}(\kappa, z, s)$, for each $\iota, \kappa, z \in \Pi$ and $t, s>0$, then the triple $(\Pi, \mathscr{F}, *)$ is called a nonArchimedean FMS.

Example 1. Let $(\Pi, d)$ be a metric space. Define $a * b=a b$ (or $a * b=\min \{a, b\}$ ) for all $a, b \in[0,1]$, and define $\mathscr{F}: \Pi^{2} \times$ $(0, \infty) \longrightarrow[0,1]$ as $\mathscr{F}(l, \kappa, t)=(t /(t+d(l, \kappa)))$ for all $\iota, \kappa \in \Pi$ and $t>0$. Then, $(\Pi, \mathscr{F}, *)$ is a FMS. We call this induced fuzzy metric by the metric $d$ the standard fuzzy metric.
Definition 3 (see $[3,8,9])$. Let $(\Pi, \mathscr{F}, *)$ be a FMS.

(i) A sequence $\left\{l_{n}\right\}$ in $\Pi$ is said to be convergent to a point $\iota \in \Pi$ whenever $\lim _{n \longrightarrow \infty} \mathscr{F}\left(\iota_{n}, l, t\right)=1$ for all $t>0$; in this case, we write $\lim _{n \longrightarrow \infty} l_{n}=\iota$

(ii) A sequence $\left\{t_{n}\right\}$ in $\Pi$ is called $\mathscr{F}$-Cauchy if, for each $0<\varepsilon<1$ and $t>0$, there exists $n_{0} \in \mathbb{N}$ such that $\mathscr{F}\left(\iota_{n}, \iota_{m}, t\right)>1-\varepsilon$, for each $n, m \geq n_{0}$

(iii) A sequence $\left\{l_{n}\right\}$ in $\Pi$ is called $G$-Cauchy if $\lim _{n \longrightarrow \infty} \mathscr{F}\left(\iota_{n}, \iota_{n+p}, t\right)=1$, for each $p \in \mathbb{N}$ and $t>0$

(iv) A FMS in which every $\mathscr{F}$-Cauchy (G-Cauchy) sequence is convergent is said to be $\mathscr{F}$-complete (G-complete)

Definition 4 (see [10]). Let $(\Pi, \mathscr{F}, *)$ be a FMS. The fuzzy metric $\mathscr{F}$ is said to be triangular if the following condition holds,

$$
\left(\frac{1}{\mathscr{F}(l, z, t)}-1\right) \leq\left(\frac{1}{\mathscr{F}(l, \kappa, t)}-1\right)+\left(\frac{1}{\mathscr{F}(\kappa, z, t)}-1\right),
$$

for all $\iota, \kappa, z \in \Pi$ and for all $t>0$.

\section{Main Results}

In this section, we give our main results of this study. Firstly, we give some notions that will be needed in the sequel.

Denote by $\Phi$ the collection of all nondecreasing right continuous functions $\eta:[0, \infty) \longrightarrow[0, \infty)$ such that $\eta(t)<t$ for all $t>0$ and $\eta^{-1}(\{0\})=\{0\}$. It is well known that $\lim _{n \longrightarrow \infty} \eta^{n}(t)=0$ for all $t>0$, where $\eta^{n}$ denotes the $n$th iterate of $\eta$.

Proposition 1 (see $[11])$. Let $(\Pi, \mathscr{F}, *)$ be a FMS. Then, $\mathscr{F}$ is a continuous function on $\Pi^{2} \times(0, \infty)$.

The notion of $\alpha$-admissibility for self-mappings in a FMS is given in [5] as follows.

Definition 5. Let $(\Pi, \mathscr{F}, *)$ be a FMS, $\alpha$ : $\Pi^{2} \times(0, \infty) \longrightarrow$ $[0, \infty)$, and $\mathbb{Q}: \Pi \longrightarrow \Pi$. $Q$ is called $\alpha$-admissible whenever, for all $t>0$,

$$
\iota, \kappa \in \Pi, \quad \alpha(l, \kappa, t) \geq 1 \Longrightarrow \alpha(\mathbb{Q} l, \mathbb{Q} \kappa, t) \geq 1 .
$$

Definition 6. Let $(\Pi, \mathscr{F}, *)$ be a FMS. Q: $\Pi \longrightarrow \Pi$ is called a generalized $\alpha-\eta$-fuzzy contractive mapping whenever there exist two functions $\eta \in \Phi$ and $\alpha: \Pi^{2} \times(0, \infty) \longrightarrow[0, \infty)$ satisfying

$$
\alpha(\iota, \kappa, t)\left(\frac{1}{\mathscr{F}(Q, Q, \mathcal{Q} \kappa, t)}-1\right) \leq \eta\left(\frac{1}{m(\iota, \kappa, t)}-1\right),
$$

for all $\iota, \kappa \in \Pi$ and $t>0$, where 


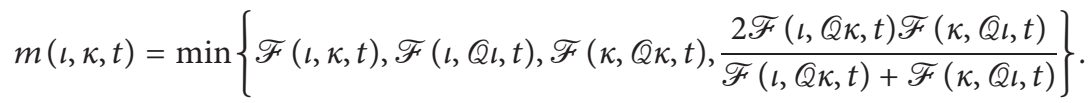

Now, we are ready to state and prove our first main result.

Theorem 1. Let $(\Pi, \mathscr{F}, *)$ be a G-complete FMS. Let $\mathscr{F}$ be triangular and $\mathbb{Q}: \Pi \longrightarrow \Pi$ be a generalized $\alpha-\eta$-fuzzy contractive mapping so that

(i) Q is $\alpha$-admissible, $=$

(ii) There exists $\iota_{0} \in \Pi$ such that $\alpha\left(\iota_{0}, Q \iota_{0}, t\right) \geq 1$, for all $t>0$

(iii) $\mathbb{Q}$ is continuous
Then, Q possesses a fixed point.

Proof. Define the sequence $\left\{l_{n}\right\}$ in $\Pi$ by $\iota_{n}=Q \iota_{n-1}$, for all $n \in \mathbb{N}$. If $\iota_{n}=\iota_{n-1}$ for some $n \in \mathbb{N}$, then $\iota^{*}=\iota_{n-1}$ is a fixed point of $Q$. Assume that $\iota_{n} \neq \iota_{n-1}$ for all $n \in \mathbb{N}$. Since $\alpha\left(\iota_{0}, \iota_{1}, t\right)=\alpha\left(\iota_{0}, Q \iota_{0}, t\right) \geq 1$ and $Q$ is $\alpha$-admissible, so we get $\alpha\left(\iota_{1}, \iota_{2}, t\right)=\alpha\left(\mathbb{Q} \iota_{0}, \iota_{1}, t\right) \geq 1$ for all $t>0$. Continuing this process we get $\alpha\left(\iota_{n-1}, \iota_{n}, t\right) \geq 1$ for all $n \in \mathbb{N}$ and for all $t>0$. By (5), for any $n \in \mathbb{N}$ and $t>0$, we have

$$
\frac{1}{\mathscr{F}\left(\iota_{n}, \iota_{n+1}, t\right)}-1=\frac{1}{\mathscr{F}\left(\mathcal{Q} \iota_{n-1}, \mathcal{Q} \iota_{n}, t\right)}-1 \leq \alpha\left(\iota_{n-1}, \iota_{n}, t\right)\left(\frac{1}{\mathscr{F}\left(\mathcal{Q} \iota_{n-1}, Q \mathcal{Q} \iota_{n}, t\right)}-1\right) \leq \eta\left(\frac{1}{m\left(\iota_{n-1}, \iota_{n}, t\right)}-1\right),
$$

where

$$
\begin{aligned}
m\left(\iota_{n-1}, \iota_{n}, t\right) & =\min \left\{\mathscr{F}\left(\iota_{n-1}, \iota_{n}, t\right), \mathscr{F}\left(\iota_{n-1}, \mathscr{Q} \iota_{n-1}, t\right), \mathscr{F}\left(\iota_{n}, \mathscr{Q} \iota_{n}, t\right), \frac{2 \mathscr{F}\left(\iota_{n-1}, \mathscr{Q} \iota_{n}, t\right) \mathscr{F}\left(\mathscr{Q} \iota_{n-1}, \iota_{n}, t\right)}{\left.\mathscr{Q} \iota_{n-1}, \iota_{n}, t\right)+\mathscr{F}\left(\iota_{n-1}, \mathscr{Q} \iota_{n}, t\right)}\right\} \\
& =\min \left\{\mathscr{F}\left(\iota_{n-1}, \iota_{n}, t\right), \mathscr{F}\left(\iota_{n}, \iota_{n+1}, t\right), \frac{2 \mathscr{F}\left(\iota_{n-1}, \iota_{n+1}, t\right)}{1+\mathscr{F}\left(\iota_{n-1}, \iota_{n+1}, t\right)}\right\} .
\end{aligned}
$$

On the contrary,

$$
\begin{aligned}
\frac{2 \mathscr{F}\left(\iota_{n-1}, \iota_{n+1}, t\right)}{1+\mathscr{F}\left(\iota_{n-1}, \iota_{n+1}, t\right)} & =\frac{2}{\left(1 / \mathscr{F}\left(\iota_{n-1}, \iota_{n+1}, t\right)\right)+1} \geq \frac{2}{\left(1 / \mathscr{F}\left(\iota_{n-1}, \iota_{n}, t\right)\right)+\left(1 / \mathscr{F}\left(\iota_{n}, l_{n+1}, t\right)\right)} \\
& \geq \min \left\{\mathscr{F}\left(\iota_{n-1}, \iota_{n}, t\right), \mathscr{F}\left(\iota_{n}, l_{n+1}, t\right)\right\} .
\end{aligned}
$$

From (8) and (9), we get $m\left(\iota_{n-1}, \iota_{n}, t\right)=\min \left\{\mathscr{F}\left(\iota_{n-1}, \iota_{n}, t\right), \mathscr{F}\left(l_{n}, l_{n+1}, t\right)\right\}$. Substituting in (7), we obtain

$$
\frac{1}{\mathscr{F}\left(\iota_{n}, \iota_{n+1}, t\right)}-1 \leq \eta\left(\frac{1}{\min \left\{\mathscr{F}\left(\iota_{n-1}, \iota_{n}, t\right), \mathscr{F}\left(\iota_{n}, \iota_{n+1}, t\right)\right\}}-1\right) .
$$

Now, if $\min \left\{\mathscr{F}\left(l_{n-1}, l_{n}, t\right), \mathscr{F}\left(l_{n}, \iota_{n+1}, t\right)\right\}=\mathscr{F}\left(\iota_{n}, l_{n+1}, t\right)$, by the property $\eta(t)<t$, then

$$
\frac{1}{\mathscr{F}\left(\iota_{n}, \iota_{n+1}, t\right)}-1 \leq \eta\left(\frac{1}{\mathscr{F}\left(\iota_{n}, \iota_{n+1}, t\right)}-1\right)<\frac{1}{\mathscr{F}\left(\iota_{n}, \iota_{n+1}, t\right)}-1,
$$

which is a contradiction. So, we have $\min \{\mathscr{F}$ $\left.\left(\iota_{n-1}, \iota_{n}, t\right), \mathscr{F}\left(\iota_{n}, \iota_{n+1}, t\right)\right\}=\mathscr{F}\left(\iota_{n-1}, \iota_{n}, t\right)$ and so

$$
\frac{1}{\mathscr{F}\left(\iota_{n}, \iota_{n+1}, t\right)}-1 \leq \eta\left(\frac{1}{\mathscr{F}\left(\iota_{n-1}, \iota_{n}, t\right)}-1\right)<\frac{1}{\mathscr{F}\left(\iota_{n-1}, \iota_{n}, t\right)}-1 .
$$

Hence, $\mathscr{F}\left(\iota_{n}, \iota_{n+1}, t\right)>\mathscr{F}\left(\iota_{n-1}, \iota_{n}, t\right)$. So, the sequence $\left\{\mathscr{F}\left(\iota_{n}, l_{n+1}, t\right)\right\}$ is strictly increasing in the interval $[0,1]$, for all $t>0$. Let $S(t)=\lim _{n \rightarrow \infty} \mathscr{F}\left(l_{n}, l_{n+1}, t\right)$, for all $t>0$. We claim that $S(t)=1$, for all $t>0$. Suppose the contradiction, $S\left(t_{0}\right)<1$, for some $t_{0}>0$. Taking limit in both sides of (12), we obtain

$$
\frac{1}{S\left(t_{0}\right)}-1 \leq \eta\left(\frac{1}{S\left(t_{0}\right)}-1\right)<\frac{1}{S\left(t_{0}\right)}-1,
$$

which is a contradiction. Thus, we have $\lim _{n \longrightarrow \infty}$ $\mathscr{F}\left(l_{n}, l_{n+1}, t\right)=1$, for all $t>0$. Now, for a fixed $p \in \mathbb{N}$, we have 


$$
\mathscr{F}\left(\iota_{n}, \iota_{n+p}, t\right) \geq \mathscr{F}\left(\iota_{n}, \iota_{n+1}, \frac{t}{p}\right) * \mathscr{F}\left(\iota_{n+1}, \iota_{n+2}, \frac{t}{p}\right) * \ldots * \mathscr{F}\left(\iota_{n+p-1}, \iota_{n+p}, \frac{t}{p}\right) \longrightarrow 1 * 1 * \ldots * 1=1
$$

as $n \longrightarrow \infty$. So, $\left\{\iota_{n}\right\}$ is a $G$-Cauchy sequence. Since $(\Pi, \mathscr{F}, *)$ is $G$-complete, there exists $\iota^{*} \in \Pi$ such that $\iota_{n} \longrightarrow \iota^{*}$, for all $t>0$. Since $Q$ is continuous, so we get Q $\iota_{n} \longrightarrow Q \iota^{*}$, for all $t>0$. Now, we have $\lim _{n \longrightarrow \infty} \mathscr{F}\left(\iota_{n+1}, \mathbb{Q} \iota^{*}, t\right)=\lim _{n \longrightarrow \infty} \mathscr{F}\left(\mathcal{Q} \iota_{n}, \mathscr{Q} \iota^{*}, t\right)=1$, for all $t>0$, that is, $\iota_{n} \longrightarrow Q \iota^{*}$. Uniqueness of the limit implies that $Q \iota^{*}=\iota^{*}$.

In the above theorem, the fixed point is unique if $\alpha(\iota, \kappa, t) \geq 1$ for all $t>0$, whenever $\iota$ and $\kappa$ are fixed points of Q.

In the following theorem, we omit the continuity condition of the mapping $Q$.

Theorem 2. We have $(\Pi, \mathscr{F}, *)$ as a G-complete FMS, $\mathscr{F}$ a triangular fuzzy set, and $Q: \Pi \longrightarrow \Pi$ as a generalized $\alpha-\eta$-fuzzy contractive mapping such that (i) Q is $\alpha$-admissible

(ii) There exists $\iota_{0} \in \Pi$ such that $\alpha\left(\iota_{0}, Q \iota_{0}, t\right) \geq 1$ for all $t>0$

(iii) For each sequence $\left\{\iota_{n}\right\}$ in $\Pi$ with $\alpha\left(\iota_{n}, \iota_{n+1}, t\right) \geq 1$, for all $n \in \mathbb{N}$ and $t>0$ such that $\iota_{n} \longrightarrow l$, we have $\alpha\left(\iota_{n}, l, t\right) \geq 1$, for all $n \in \mathbb{N}$ and $t>0$

Then, Q admits a fixed point.

Proof. Following the proof of Theorem 1, we obtain a sequence $\left\{l_{n}\right\}$ in $\Pi$ which is G-Cauchy, $\alpha\left(t_{n}, \iota_{n+1}, t\right) \geq 1$, for all $n \in \mathbb{N}$, and there exists $\iota^{*} \in \Pi$ such that $\iota_{n} \longrightarrow \iota^{*}$. From condition (iii), we get $\alpha\left(\iota_{n}, \iota^{*}, t\right) \geq 1$, for all $n \in \mathbb{N}$ and $t>0$. Now, we have

$$
\begin{aligned}
\frac{1}{\mathscr{F}\left(Q \iota^{*}, \iota^{*}, t\right)}-1 & \leq\left(\frac{1}{\mathscr{F}\left(Q \iota^{*}, Q \iota_{n}, t\right)}-1\right)+\left(\frac{1}{\mathscr{F}\left(\iota_{n+1}, \iota^{*}, t\right)}-1\right) \\
& \leq \alpha\left(\iota_{n}, \iota^{*}, t\right)\left(\frac{1}{\mathscr{F}\left(Q \iota_{n}, Q \iota^{*}, t\right)}-1\right)+\left(\frac{1}{\mathscr{F}\left(\iota_{n+1}, \iota^{*}, t\right)}-1\right) \leq \eta\left(\frac{1}{m\left(\iota_{n}, \iota^{*}, t\right)}-1\right)+\left(\frac{1}{\mathscr{F}\left(\iota_{n+1}, \iota^{*}, t\right)}-1\right) .
\end{aligned}
$$

On the contrary,

$$
\begin{aligned}
\mathscr{F}\left(\iota^{*}, \mathscr{Q} \iota^{*}, t\right) \geq m\left(\iota_{n}, \iota^{*}, t\right) & =\min \left\{\mathscr{F}\left(\iota_{n}, \iota^{*}, t\right), \mathscr{F}\left(\iota_{n}, \mathscr{Q} \iota_{n}, t\right), \mathscr{F}\left(\iota^{*}, \mathscr{Q} \iota^{*}, t\right), \frac{2 \mathscr{F}\left(\iota_{n}, \mathscr{Q} \iota^{*}, t\right) \mathscr{F}\left(\mathscr{Q} \iota_{n}, \iota^{*}, t\right)}{\mathscr{F}\left(\iota_{n}, \mathscr{Q} \iota^{*}, t\right)+\mathscr{F}\left(\mathscr{Q} \iota_{n}, \iota^{*}, t\right)}\right\} \\
& =\min \left\{\mathscr{F}\left(\iota_{n}, \iota^{*}, t\right), \mathscr{F}\left(\iota_{n}, \iota_{n+1}, t\right), \mathscr{F}\left(\iota^{*}, \mathscr{Q} \iota^{*}, t\right), \frac{2 \mathscr{F}\left(\iota_{n}, \mathscr{Q} \iota^{*}, t\right) \mathscr{F}\left(\iota_{n+1}, \iota^{*}, t\right)}{\mathscr{F}\left(\iota_{n}, \mathbb{Q} \iota^{*}, t\right)+\mathscr{F}\left(\iota_{n+1}, \iota^{*}, t\right)}\right\},
\end{aligned}
$$

where

$$
\begin{aligned}
\left.\frac{2 \mathscr{F}\left(\iota_{n}, Q \iota^{*}, t\right) \mathscr{F}\left(\iota_{n+1}, \iota^{*}, t\right)}{\mathscr{F}\left(\iota_{n}, Q \iota^{*}, t\right)+\mathscr{F}\left(\iota_{n+1}, \iota^{*}, t\right)}\right\} & =\frac{2}{\left(1 / \mathscr{F}\left(t_{n+1}, \iota^{*}, t\right)\right)+\left(1 / \mathscr{F}\left(t_{n}, \mathbb{Q} \iota^{*}, t\right)\right)} \\
& \geq \frac{2}{\left(1 / \mathscr{F}\left(t_{n+1}, \iota^{*}, t\right)\right)+\left(1 / \mathscr{F}\left(t_{n}, \iota^{*}, t\right)\right)+\left(1 / \mathscr{F}\left(\iota^{*}, Q \iota^{*}, t\right)\right)-1} \\
& \longrightarrow \frac{2}{1+\left(1 / \mathscr{F}\left(\iota^{*}, Q \iota^{*}, t\right)\right)} \\
& \geq \frac{2}{\left(1 / \mathscr{F}\left(\iota^{*}, \mathscr{Q} \iota^{*}, t\right)\right)+\left(1 / \mathscr{F}\left(\iota^{*}, \mathscr{Q} \iota^{*}, t\right)\right)}=\mathscr{F}\left(\iota^{*}, Q \iota^{*}, t\right) .
\end{aligned}
$$


Thus, taking limit on both sides of (16) as $n \longrightarrow \infty$, we get $\lim _{n \longrightarrow \infty} m\left(\iota_{n}, \iota^{*}, t\right)=\mathscr{F}\left(\iota^{*}, Q_{\iota} \iota^{*}, t\right)$. Now, taking limit on both sides of $(15)$ as $n \longrightarrow \infty$ and from right continuity of $\eta$, we obtain $\left(1 / \mathscr{F}\left(\mathscr{Q} \iota^{*}, \iota^{*}, t\right)\right)-1 \leq \eta\left(\left(1 / \mathscr{F}\left(\mathbb{Q} \iota^{*}, \iota^{*}, t\right)\right)-1\right)$. This implies $\left(1 / \mathscr{F}\left(\mathbb{Q} \iota^{*}, \iota^{*}, t\right)\right)-1=0 . \quad$ Therefore, $\mathscr{F}\left(\mathbb{Q} \iota^{*}, \iota^{*}, t\right)=1$ and so $\mathbb{Q} \iota^{*}=\iota^{*}$.

Remark 1. Since $m(l, \kappa, t) \leq \mathscr{F}(l, \kappa, t) \quad$ implies $((1 / \mathscr{F}(\iota, \kappa, t))-1) \leq((1 / m(\iota, \kappa, t))-1)$, for all $\iota, \kappa \in \Pi$ and $t>0$, thus any $\alpha-\eta$-fuzzy contractive mapping in the sense of [5] which is a generalized $\alpha-\eta$-fuzzy contractive mapping. Therefore, Theorems 1 and 2 are generalizations of Theorems 3.5 and 3.6 in [5], respectively.

The following example shows that Theorem 2 is a real generalization of Theorem 3.6 in [5].

Example 2. Let

$$
\begin{aligned}
& A_{1}=\left\{\frac{m}{n}: m=0,1,3,9, \ldots, n=1,4, \ldots, 3 k+1, \ldots\right\}, \\
& A_{2}=\left\{\frac{m}{n}: m=1,3,9, \ldots, n=2,5, \ldots, 3 k+2, \ldots\right\}, \\
& A_{3}=\{2 k: k \in \mathbb{N}\},
\end{aligned}
$$

and $\Pi=A_{1} \cup A_{2} \cup A_{3}$. Let $a * b=a b$, for all $a, b \in[0,1]$, and $\mathscr{F}(\iota, \kappa, t)=(t / t+|\iota-\kappa|)$, for all $\iota, \kappa \in \Pi$ and $t>0$. Define Q: $\Pi \longrightarrow \Pi$ by

$$
Q \iota= \begin{cases}\frac{3 \iota}{11}, & \iota \in A_{1}, \\ \frac{\iota}{8}, & \iota \in A_{2}, \\ 2 \iota, & \iota \in A_{3},\end{cases}
$$

and $\alpha: \Pi^{2} \times(0, \infty) \longrightarrow[0, \infty)$ by

$$
\alpha(\iota, \kappa, t)= \begin{cases}1, & \iota, \kappa \in A_{1} \cup A_{2}, \\ 0, & \text { otherwise }\end{cases}
$$

If $\iota, \kappa \in A_{1}$, then

$$
\left(\frac{1}{\mathscr{F}(\mathscr{Q} \iota, \mathbb{Q} \kappa, t)}-1\right)=\frac{|(3 \iota / 11)-(3 \kappa / 11)|}{t}=\frac{3}{11} \frac{|\iota-\kappa|}{t}=\frac{3}{11}\left(\frac{1}{\mathscr{F}(\iota, \kappa, t)}-1\right) \leq \frac{6}{11}\left(\frac{1}{m(\iota, \kappa, t)}-1\right) .
$$

If $\iota, \kappa \in A_{2}$, then

$$
\left(\frac{1}{\mathscr{F}(\mathscr{Q} \iota, Q \mathcal{Q} \kappa, t)}-1\right)=\frac{|(\iota / 8)-(\kappa / 8)|}{t}=\frac{1}{8} \frac{|\iota-\kappa|}{t}=\frac{1}{8}\left(\frac{1}{\mathscr{F}(\iota, \kappa, t)}-1\right) \leq \frac{6}{11}\left(\frac{1}{m(\iota, \kappa, t)}-1\right) .
$$

If $\iota, \kappa \in A_{3}$, then $\alpha(\iota, \kappa, t)=0$, and clearly (8) holds true.

So, if $\iota>(11 / 24) \kappa$, then

If $\iota \in A_{1}$ and $\kappa \in A_{2}$, then

$$
\left(\frac{1}{\mathscr{F}(\mathbb{Q} \iota, \mathbb{Q} \kappa, t)}-1\right)=\frac{|(3 \iota / 11)-(\kappa / 8)|}{t}=\frac{3}{11} \frac{|\iota-(11 / 24) \kappa|}{t} \text {. }
$$

$$
\begin{aligned}
\left(\frac{1}{\mathscr{F}(Q \iota, Q \mathcal{Q} \kappa, t)}-1\right) & =\frac{3}{11} \frac{(\iota-(11 / 24) \kappa)}{t} \leq \frac{3}{11} \frac{(\iota-(1 / 8) \kappa)}{t}=\frac{6}{11}\left[\frac{1}{2}\left(\frac{1}{\mathscr{F}(\iota, \mathbb{Q} \kappa, t)}-1\right)\right] \\
& =\frac{6}{11}\left[\frac{1}{2}\left(\frac{1}{\mathscr{F}(\iota, \mathscr{Q} \kappa, t)}+1\right)-1\right] \leq \frac{6}{11}\left[\frac{1}{2}\left(\frac{1}{\mathscr{F}(\iota, \mathscr{Q} \kappa, t)}+\frac{1}{\mathscr{F}(\kappa, \mathscr{Q} \iota, t)}\right)-1\right] \\
& =\frac{6}{11}\left[\frac{1}{((2 \mathscr{F}(\iota, \mathbb{Q} \kappa, t) \mathscr{F}(\kappa, \mathbb{Q} \iota, t)) /(\mathscr{F}(l, \mathbb{Q} \kappa, t)+\mathscr{F}(\kappa, \mathbb{Q} \iota, t)))}-1\right] \leq \frac{6}{11}\left(\frac{1}{m(l, \kappa, t)}-1\right),
\end{aligned}
$$


and if $\iota<(11 / 24) \kappa$, then

$$
\left(\frac{1}{\mathscr{F}(Q \iota, Q \kappa, t)}-1\right)=\frac{3}{11} \frac{((11 / 24) \kappa-\iota)}{t} \leq \frac{3}{11} \frac{(\kappa-\iota)}{t} \leq \frac{3}{11}\left(\frac{1}{\mathscr{F}(\iota, \kappa, t)}-1\right) \leq \frac{6}{11}\left(\frac{1}{m(\iota, \kappa, t)}-1\right)
$$

We see that $((1 / \mathscr{F}(\mathscr{Q} \iota, Q \mathcal{Q}, t))-1) \leq(6 / 11)((1 / m$ $(\iota, \kappa, t))-1)$, for all $\iota, \kappa \in A_{1} \cup A_{2}$. So, by definition of the mapping $\alpha$, we get $\alpha(l, \kappa, t)((1 / \mathscr{F}(\mathscr{Q} l, \mathbb{Q} \kappa, t))-1) \leq$ $(6 / 11)((1 / m(l, \kappa, t))-1)$ for all $l, \kappa \in \Pi$. Thus, $Q$ is a generalized $\alpha-\eta$-fuzzy contractive mapping with $\eta(t)=(6 / 11) t$.
Also, for $\iota_{0}=1$, we have $\alpha\left(\iota_{0}, Q \iota_{0}, t\right)=\alpha(1,(3 / 11), t)=1$. It is easy to check that $Q$ is $\alpha$-admissible and the condition (iii) in Theorem 2 holds. So, by Theorem 2, Q possesses a fixed point. Here, $Q 20=0$. Now, let $\iota=1, \kappa=(27 / 29)$. Then, we have

$$
\begin{aligned}
\alpha(l, \kappa, t)\left(\frac{1}{\mathscr{F}(\mathbb{Q} \iota, \mathbb{Q} \kappa, t)}-1\right) & =\frac{|(3 / 11)-(1 / 8)(27 / 29)|}{t}=\frac{399}{2552 t}>\frac{2}{29 t}=\frac{|1-(27 / 29)|}{t} \\
& =\left(\frac{1}{\mathscr{F}(l, \kappa, t)}-1\right)>\eta\left(\frac{1}{\mathscr{F}(l, \kappa, t)}-1\right) .
\end{aligned}
$$

Thus, $\mathbb{Q}$ is not an $\alpha-\eta$-fuzzy contractive mapping. Therefore, the main result of [5] is not applicable in this example.

Let $(\Pi, \leq)$ be a poset. A mapping $Q$ : $\Pi \longrightarrow \Pi$ is called nondecreasing if $\iota \leq \kappa$ implies $Q \iota \leq \widehat{Q} \kappa$, for all $\iota, \kappa \in \Pi$.
Definition 7 . Let $(\Pi, \leq)$ be a poset and $(\Pi, \mathscr{F}, *)$ be a FMS. We say that $Q: \Pi \longrightarrow \Pi$ is a generalized fuzzy ordered $\eta$ -contractive mapping whenever there exists $\eta \in \Phi$ such that

$$
\left(\frac{1}{\mathscr{F}(Q \ell, Q \kappa, t)}-1\right) \leq \eta\left(\frac{1}{m(\iota, \kappa, t)}-1\right)
$$

for all $\iota, \kappa \in \Pi$ with $\iota \leq \kappa$ and $t>0$, where

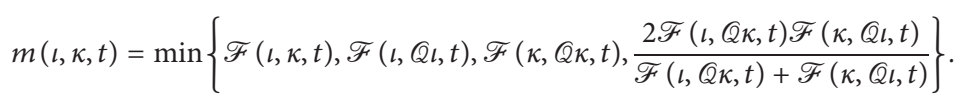

Corollary 1. Let $(\Pi, \leq)$ be a poset and $(\Pi, \mathscr{F}, *)$ be a $G$-complete FMS. Let $\mathscr{F}$ be triangular and $Q: \Pi \longrightarrow \Pi$ be a generalized fuzzy ordered $\eta$-contractive such that

(i) Q is nondecreasing

(ii) There exists $\iota_{0} \in \Pi$ such that $\iota_{0} \leq \mathbb{Q} \iota_{0}$

(iii) $Q$ is continuous

Then, Q admits a fixed point.

Proof. Define the function $\alpha: \Pi^{2} \times(0, \infty) \longrightarrow[0, \infty)$ by $\alpha(\iota, \kappa, t)=1$ if $\iota \leq \kappa$ and $\alpha(\iota, \kappa, t)=0$ otherwise. Then, apply Theorem 1.

Corollary 2. Let $(\Pi, \leq)$ be a poset and $(\Pi, \mathscr{F}, *)$ be a G-complete FMS. Let $\mathscr{F}$ be triangular and Q: $\Pi \longrightarrow \Pi$ be a generalized fuzzy ordered $\eta$-contractive mapping such that

(i) $\mathbb{Q}$ is nondecreasing (ii) There exists $\iota_{0} \in \Pi$ such that $\iota_{0} \leq \mathbb{Q} \iota_{0}$

(iii) For each nondecreasing sequence $\left\{\iota_{n}\right\}$ in $\Pi$ which $\iota_{n} \longrightarrow \iota$, we have $\iota_{n} \leq \iota$, for all $n \in \mathbb{N}$

Then, Q possesses a fixed point.

Proof. Define the function $\alpha: \Pi^{2} \times(0, \infty) \longrightarrow[0, \infty)$ by $\alpha(\iota, \kappa, t)=1$ if $\iota \leq \kappa$ and $\alpha(\iota, \kappa, t)=0$ otherwise. Then, apply Theorem 2.

Using equivalent conditions from [12], we can find also some equivalent results for Theorems 1 and 2 .

Proposition 2. Let $(\Pi, \mathscr{F}, *)$ be a G-complete FMS. Let $\mathscr{F}$ be triangular and $Q: \Pi \longrightarrow \Pi$ be a mapping such that there exist a mapping $\alpha: \Pi^{2} \times(0, \infty) \longrightarrow[0, \infty)$, a lower semicontinuous function $\zeta:[0, \infty) \longrightarrow[0, \infty)$ and a function $\eta:[0, \infty) \longrightarrow[0, \infty) \quad$ with $\eta^{-1}\{0\}=\{0\} \quad$ and $\liminf _{t \rightarrow \infty} \eta(t)>0$ such that

$$
\zeta\left(\alpha(\iota, \kappa, t)\left(\frac{1}{\mathscr{F}(\mathscr{Q} \iota, \mathbb{Q} \kappa, t)}-1\right)\right) \leq \zeta\left(\frac{1}{m(\iota, \kappa, t)}-1\right)-\eta\left(\frac{1}{m(\iota, \kappa, t)}-1\right)
$$


for all $\iota, \kappa \in \Pi$ and $t>0$. Moreover, assume that

(i) $Q$ is $\alpha$-admissible

(ii) There exists $\iota_{0} \in \Pi$ such that $\alpha\left(\iota_{0}, Q \iota_{0}, t\right) \geq 1$, for all $t>0$

(iii) $\mathbb{Q}$ is continuous or for each sequence $\left\{l_{n}\right\}$ in $\Pi$ with $\alpha\left(\iota_{n}, l_{n+1}, t\right) \geq 1$, for all $n \in \mathbb{N}$ and $t>0$, for which $\iota_{n} \longrightarrow l$, and we have $\alpha\left(\iota_{n}, l, t\right) \geq 1$ for all $n \in \mathbb{N}$ and $t>0$

Then, $\mathbb{Q}$ admits a fixed point.

Proposition 3. Let $(\Pi, \mathscr{F}, *)$ be a G-complete FMS. Let $\mathscr{F}$ be triangular and $\mathbb{Q}: \Pi \longrightarrow \Pi$ be a mapping such that there exist a mapping $\alpha: \Pi^{2} \times(0, \infty) \longrightarrow[0, \infty)$, a continuous from right function $\zeta:[0, \infty) \longrightarrow[0, \infty)$ with $\Sigma_{n=1}^{\infty} \zeta^{n}(t)<\infty$ for all $t>0$ and a function $\eta:[0, \infty) \longrightarrow[0, \infty)$ with $\eta(t)<t$ for all $t>0$ such that

$$
\zeta\left(\alpha(\iota, \kappa, t)\left(\frac{1}{\mathscr{F}(\mathscr{Q} \iota, \mathbb{Q} \kappa, t)}-1\right)\right) \leq \eta\left(\zeta\left(\frac{1}{m(\iota, \kappa, t)}-1\right)\right),
$$

for all $\iota, \kappa \in \Pi$ and $t>0$. Moreover, let

(i) $\mathbb{Q}$ is $\alpha$-admissible

(ii) There exists $\iota_{0} \in \Pi$ such that $\alpha\left(\iota_{0}, \mathcal{Q} \iota_{0}, t\right) \geq 1$ for all $t>0$

(iii) Q is continuous or for each sequence $\left\{l_{n}\right\}$ in $\Pi$ with $\alpha\left(t_{n}, l_{n+1}, t\right) \geq 1$ for all $n \in \mathbb{N}$ and $t>0$ for which $\underset{t>0}{\iota_{n}} \longrightarrow$, and we have $\alpha\left(\iota_{n}, l, t\right) \geq 1$ for all $n \in \mathbb{N}$ and

Then, $t>0$ possesses a fixed point.

\section{Generalized $\beta-\zeta$-Fuzzy Contractive Mappings}

In this section, we introduce generalized $\beta$ - $\zeta$-fuzzy contractive mappings and prove existence of fixed point for such mappings. Denote by $\Psi$ the collection of all nondecreasing left continuous functions $\zeta:[0,1] \longrightarrow[0,1]$ such that $\zeta(r)>r$, for all $r \in(0,1)$. It is well known that $\lim _{n \longrightarrow \infty} \zeta^{n}(r)=1$ and $\zeta(1)=1$, for all $r \in(0,1)$, where $\zeta^{n}$ denotes the $n$th iterate of $\zeta$.

Definition 8 (see [5]). Let $(\Pi, \mathscr{F}, *)$ be a FMS, $\beta: \Pi^{2} \times(0, \infty) \longrightarrow[0, \infty)$, and $\mathbb{Q}: \Pi \longrightarrow \Pi$ be a mapping. It is called that $\mathbb{Q}$ is $\beta$-admissible whenever, for all $t>0$,

$$
\iota, \kappa \in \Pi, \quad \beta(\iota, \kappa, t) \leq 1 \Longrightarrow \beta(\mathbb{Q} \iota, \mathbb{Q} \kappa, t) \leq 1 .
$$

Definition 9. Let $(\Pi, \mathscr{F}, *)$ be a FMS. We say that Q: $\Pi \longrightarrow \Pi$ is a generalized $\beta$ - $\zeta$-fuzzy contractive mapping whenever there exist two functions $\zeta \in \Psi$ and $\beta: \Pi^{2} \times(0, \infty) \longrightarrow[0, \infty)$ satisfying

$$
\beta(l, \kappa, t) \mathscr{F}(\mathbb{Q} l, \mathbb{Q} \kappa, t) \geq \zeta(m(l, \kappa, t)),
$$

for all $\iota, \kappa \in \Pi$ and $t>0$, where

$$
m(l, \kappa, t)=\min \left\{\mathscr{F}(\iota, \kappa, t), \mathscr{F}(\iota, \mathscr{Q} \iota, t), \mathscr{F}(\kappa, \mathbb{Q} \kappa, t), \frac{2 \mathscr{F}(\iota, \mathbb{Q} \kappa, t) \mathscr{F}(\kappa, \mathbb{Q} \iota, t)}{\mathscr{F}(\iota, \mathscr{Q} \kappa, t)+\mathscr{F}(\kappa, \mathbb{Q} \iota, t)}\right\} .
$$

Theorem 3. Let $(\Pi, \mathscr{F}, *)$ be a $\mathscr{F}$-complete non-Archimedean FMS and $\mathbb{Q}: \Pi \longrightarrow \Pi$ be a generalized $\beta$ - $\zeta$-fuzzy contractive mapping so that

(i) $\mathbb{Q}$ is $\beta$-admissible

(ii) There exists $\iota_{0} \in \Pi$ such that $\beta\left(\iota_{0}, Q \iota_{0}, t\right) \leq 1$ for all $t>0$

(iii) For each sequence $\left\{\iota_{n}\right\}$ in $\Pi$ with $\beta\left(\iota_{n}, \iota_{n+1}, t\right) \leq 1$, for all $n \in \mathbb{N}$ and $t>0$, we have $\beta\left(t_{m}, l_{n}, t\right) \leq 1$, for all $m, n \in \mathbb{N}$, with $n>m$ and $t>0$

(iv) For each sequence $\left\{l_{n}\right\}$ in $\Pi$ with $\beta\left(\iota_{n}, l_{n+1}, t\right) \leq 1$, for all $n \in \mathbb{N}$ and $t>0$, in which $\iota_{n} \longrightarrow l$, and we have $\beta\left(\iota_{n}, l, t\right) \leq 1$, for all $n \in \mathbb{N}$ and $t>0$
Then, $\mathbb{Q}$ admits a fixed point.

Proof. Define the sequence $\left\{l_{n}\right\}$ in $\Pi$ by $\iota_{n}=\mathbb{Q} l_{n-1}$, for all $n \in \mathbb{N}$. If $\iota_{n}=\iota_{n-1}$ for some $n \in \mathbb{N}$, then $\iota^{*}=\iota_{n-1}$ is a fixed point of $Q$. Assume that $\iota_{n} \neq \iota_{n-1}$, for all $n \in \mathbb{N}$. Since $\beta\left(\iota_{0}, \iota_{1}, t\right)=\beta\left(\iota_{0}, \mathbb{Q} \iota_{0}, t\right) \leq 1$ and $\mathbb{Q}$ is $\beta$-admissible, so we get $\beta\left(\iota_{1}, l_{2}, t\right)=\beta\left(Q \iota_{0}, Q \iota_{1}, t\right) \leq 1$ for all $t>0$. Continuing this process, we get $\beta\left(l_{n-1}, l_{n}, t\right) \leq 1$ for all $n \in \mathbb{N}$ and for all $t>0$. By (32), for any $n \in \mathbb{N}$ and $t>0$, we have

$$
\mathscr{F}\left(\iota_{n}, l_{n+1}, t\right)=\mathscr{F}\left(\mathscr{Q} l_{n-1}, \mathbb{Q} l_{n}, t\right) \geq \beta\left(l_{n-1}, l_{n}, t\right) \mathscr{F}\left(\mathscr{Q} l_{n-1}, \mathscr{Q} l_{n}, t\right) \geq \zeta\left(m\left(l_{n-1}, l_{n}, t\right)\right),
$$

where, as in the proof of Theorem 1, we have

$$
m\left(l_{n-1}, l_{n}, t\right)=\min \left\{\mathscr{F}\left(l_{n-1}, l_{n}, t\right), \mathscr{F}\left(l_{n}, l_{n+1}, t\right)\right\} .
$$

Substituting in (34), we obtain

$$
\mathscr{F}\left(\iota_{n}, l_{n+1}, t\right) \geq \zeta\left(\min \left\{\mathscr{F}\left(l_{n-1}, l_{n}, t\right), \mathscr{F}\left(l_{n}, l_{n+1}, t\right)\right\}\right) .
$$



then

Now, if $\min \left\{\mathscr{F}\left(\iota_{n-1}, \iota_{n}, t\right), \mathscr{F}\left(\iota_{n}, \iota_{n+1}, t\right)\right\}=\mathscr{F}\left(\iota_{n}, \iota_{n+1}, t\right)$,

$$
\mathscr{F}\left(l_{n}, l_{n+1}, t\right) \geq \zeta\left(\mathscr{F}\left(l_{n}, l_{n+1}, t\right)\right)>\mathscr{F}\left(\iota_{n}, l_{n+1}, t\right),
$$

which is a contradiction. So, we have

$$
\min \left\{\mathscr{F}\left(l_{n-1}, l_{n}, t\right), \mathscr{F}\left(l_{n}, l_{n+1}, t\right)\right\}=\mathscr{F}\left(l_{n-1}, l_{n}, t\right),
$$

so

$$
\mathscr{F}\left(\iota_{n}, \iota_{n+1}, t\right) \geq \zeta\left(\mathscr{F}\left(\iota_{n-1}, \iota_{n}, t\right)\right) .
$$

From (39), we obtain

$$
\mathscr{F}\left(\iota_{n}, \iota_{n+1}, t\right) \geq \zeta^{n}\left(\mathscr{F}\left(l_{0}, \iota_{1}, t\right)\right) \longrightarrow 1,
$$

as $n \longrightarrow \infty$. So, we have $\lim _{n \longrightarrow \infty} \mathscr{F}\left(l_{n}, l_{n+1}, t\right)=1$, for all $t>0$. Now, if $\left\{l_{n}\right\}$ is not an $\mathscr{F}$-Cauchy sequence, then there are $\varepsilon \in(0,1), t>0$ and $k_{0} \in \mathbb{N}$ such that, for each $k \in \mathbb{N}$ with $k \geq k_{0}$, there exist $m(k), n(k) \in \mathbb{N}$ with $m(k)>n(k) \geq k$ satisfying

$$
\mathscr{F}\left(l_{m(k)}, l_{n(k)}, t\right) \leq 1-\varepsilon, \beta\left(l_{m(k)}, l_{n(k)}, t\right) \leq 1 .
$$

Let, for each $k, m(k)$ be the least positive integer exceeding $n(k)$ satisfying the above property, that is,

$$
\mathscr{F}\left(l_{m(k)-1}, \iota_{n(k)}, t\right)>1-\varepsilon, \mathscr{F}\left(\iota_{m(k)}, \iota_{n(k)}, t\right) \leq 1-\varepsilon .
$$

Now, we have

$$
1-\varepsilon \geq \mathscr{F}\left(l_{m(k)-1}, l_{n(k)}, t\right) \geq \mathscr{F}\left(l_{m(k)-1}, l_{n(k)}, t\right) * \mathscr{F}\left(l_{m(k)-1}, l_{m(k)}, t\right) \geq(1-\varepsilon) \mathscr{F}\left(l_{m(k)-1}, l_{m(k)}, t\right) .
$$

Thus, $\lim _{k \longrightarrow \infty} \mathscr{F}\left(l_{m(k)-1}, l_{n(k)}, t\right)=1-\varepsilon$, for all $t>0$. Also, we have

$$
\begin{aligned}
\mathscr{F}\left(l_{m(k)-1}, l_{n(k)}, t\right) & \geq \mathscr{F}\left(l_{m(k)-1}, l_{m(k)+1}, t\right) * \mathscr{F}\left(l_{m(k)-1}, l_{n(k)+1}, t\right) * \mathscr{F}\left(l_{n(k)+1}, l_{n(k)}, t\right) \\
& \geq \mathscr{F}\left(l_{m(k)-1}, l_{m(k)+1}, t\right) * \beta\left(l_{m(k)-1}, l_{n(k)+1}, t\right) \mathscr{F}\left(\mathscr{Q} l_{m(k)}, \mathscr{Q} l_{n(k)}, t\right) * \mathscr{F}\left(\iota_{n(k)+1}, l_{n(k)}, t\right) \\
& \geq \mathscr{F}\left(l_{m(k)-1}, l_{m(k)+1}, t\right) * \zeta\left(m\left(l_{m(k)}, l_{n(k)}, t\right)\right) * \mathscr{F}\left(l_{n(k)+1}, l_{n(k)}, t\right) .
\end{aligned}
$$

On the contrary,

$$
\begin{aligned}
& \mathscr{F}\left(l_{m(k)}, l_{n(k)}, t\right) \geq m\left(l_{m(k)}, l_{n(k)}, t\right)=\min \left\{\mathscr{F}\left(l_{m(k)}, l_{n(k)}, t\right), \mathscr{F}\left(l_{m(k)}, l_{m(k+1)}, t\right), \mathscr{F}\left(l_{n(k)}, l_{n(k)+1}, t\right),\right. \\
& \left.\frac{2 \mathscr{F}\left(l_{m(k)}, l_{n(k)+1}, t\right) \mathscr{F}\left(l_{m(k)+1}, l_{n(k)}, t\right)}{\mathscr{F}\left(l_{m(k)}, l_{n(k)+1}, t\right)+\mathscr{F}\left(l_{m(k)+1}, l_{n(k)}, t\right)}\right\} \\
& =\min \left\{\mathscr{F}\left(l_{m(k)}, \iota_{n(k)}, t\right), \mathscr{F}\left(\iota_{m(k)}, l_{m(k)+1}, t\right), \mathscr{F}\left(l_{n(k)}, l_{n(k)+1}, t\right), \frac{2}{\left(1 / \mathscr{F}\left(l_{m(k)}, l_{n(k)+1}, t\right)\right)+\left(1 / \mathscr{F}\left(\iota_{m(k)}, l_{n(k)}, t\right)\right)}\right\} \\
& \geq \min \left\{\mathscr{F}\left(l_{m(k)}, l_{n(k)}, t\right), \mathscr{F}\left(l_{m(k)}, l_{m(k)+1}, t\right), \mathscr{F}\left(l_{n(k)}, \iota_{n(k)+1}, t\right),\right. \\
& \left.\frac{2}{\left(1 / \mathscr{F}\left(l_{m(k)}, l_{n(k)}, t\right) * \mathscr{F}\left(l_{n(k)}, l_{n(k)+1}, t\right)\right)+\left(1 / \mathscr{F}\left(l_{m(k)+1}, l_{m(k)}, t\right) * \mathscr{F}\left(l_{m(k)}, l_{n(k)}, t\right)\right)}\right\} .
\end{aligned}
$$

Taking the limit in the above inequality, we obtain $1-\varepsilon \geq \lim _{k \rightarrow \infty} m\left(l_{m(k)}, l_{n(k)}, t\right) \geq \min \{1-\varepsilon, 1,1,1-\varepsilon\}=1-\varepsilon$,

which implies

$$
\lim _{k \rightarrow \infty} m\left(\iota_{m(k)}, \iota_{n(k)}, t\right)=1-\varepsilon .
$$

Taking limit in (44) and from left continuity of $\zeta$, we obtain

$$
1-\varepsilon \geq \zeta(1-\varepsilon)>1-\varepsilon
$$

This contradiction shows that $\left\{l_{n}\right\}$ is a $\mathscr{F}$-Cauchy sequence. Let $\iota_{n} \longrightarrow \iota^{*}$. Then, from assumption (iv), $\beta\left(\iota_{n}, l^{*}, t\right) \leq 1$, for all $n \in \mathbb{N}$ and $t>0$. Now, we have 


$$
\begin{aligned}
\mathscr{F}\left(\mathscr{Q} \iota^{*}, \iota^{*}, t\right) & \geq \mathscr{F}\left(\mathbb{Q} \iota_{n}, \mathscr{Q} \iota^{*}, t\right) * \mathscr{F}\left(\iota_{n+1}, \iota^{*}, t\right) \geq \beta\left(\iota_{n}, \iota^{*}, t\right) \mathscr{F}\left(\mathbb{Q} \iota_{n}, \mathscr{Q} \iota^{*}, t\right) * \mathscr{F}\left(\iota_{n+1}, \iota^{*}, t\right) \\
& \geq \zeta\left(m\left(\iota_{n}, \iota^{*}, t\right)\right) * \mathscr{F}\left(\iota_{n+1}, \iota^{*}, t\right) .
\end{aligned}
$$

On the contrary, as in the proof of Theorem 2, we have

$$
\lim _{n \longrightarrow \infty} m\left(\iota_{n}, \iota^{*}, t\right)=\mathscr{F}\left(Q \iota^{*}, \iota^{*}, t\right) .
$$

Taking limit in (49) and from left continuity of $\zeta$, we obtain

$$
\mathscr{F}\left(\mathbb{Q} \iota^{*}, \iota^{*}, t\right) \geq \zeta\left(\mathscr{F}\left(\mathbb{Q} \iota^{*}, \iota^{*}, t\right)\right),
$$

which implies $\mathscr{F}\left(\mathbb{Q} \iota^{*}, \iota^{*}, t\right)=1$, so $\mathbb{Q} \iota^{*}=\iota^{*}$.

Remark 2. Since $\mathscr{F}(\iota, \kappa, t) \geq m(\iota, \kappa, t)$, for all $\iota, \kappa \in \Pi$ and $t>0$, thus any $\beta$ - $\zeta$-fuzzy contractive mapping in the sense of [5] which is a generalized $\beta$ - $\zeta$-fuzzy contractive mapping. Therefore, Theorem 3 is a generalization of Theorem 4.4 in [5].

\section{Application}

This section is devoted to the application for the uniqueness of the solution of an integral equation. Consider the integral equation,

$$
\iota(r)=g(r)+\int_{0}^{r} F(r, s, \iota(s) \mathrm{d} s), \quad \text { for all } r \in[0, I], I>0,
$$

and Banach space $C([0, I], \mathbb{R})$ of all continuous functions defined on $[0, I]$ equipped with supremum norm,

$$
\|\iota\|=\sup _{r \in[0,1]}|\iota(r)|, \quad \iota \in C([0, I], \mathbb{R}),
$$

with induced metric

$$
d(\iota, \kappa)=\sup _{r \in[0, I]}|\iota(r)-\kappa(r)| .
$$

Now, consider the FMS with product $t$-norm as

$$
\mathscr{F}(\iota, \kappa, t)=\frac{t}{t+d(\iota, \kappa)}, \quad \text { for all } \iota, \kappa \in C([0, I], \mathbb{R}), t>0 \text {. }
$$

According to [3], standard FMS and the corresponding metric space have same topologies. So, FMS defined in (55) is complete.

Theorem 6. Consider the integral operator $\mathbb{Q}$ in $C([0, I], \mathbb{R})$ as

$$
Q_{\iota \iota}(r)=g(r)+\int_{0}^{r} F(r, s, \iota(s) \mathrm{d} s),
$$

where $F$ satisfies the following conditions: there exists $f:[0, I] \times[0, I] \longrightarrow[0, \infty)$ such that $f \in L^{1}([0, I], \mathbb{R})$ and for all $\iota, \kappa \in C([0, I], \mathbb{R}), r, s \in[0, I]$, we have

$$
|F(s, r, \iota(r))-F(s, r, \kappa(r))| \leq f(r, s) \max \left\{|\iota(s)-\kappa(s)|,|\iota(s)-\mathbb{Q} \kappa(s)|,|\kappa(s)-\mathscr{Q} \kappa(s)|, \frac{2 \mid \iota(s)-\mathscr{Q} \kappa(s) \| \kappa(s))-\mathscr{Q} \iota(s) \mid}{|\iota(s)-\mathscr{Q} \kappa(s)|+\iota(s)-\kappa(s) \mid}\right\},
$$

where

$$
\sup _{r \in[0,1]} \int_{0}^{r} f(r, s) \mathrm{d} s \leq \lambda<1 .
$$

Then, the integral equation (52) possesses a unique solution.

Proof. Let $\iota, \kappa \in C([0, I], \mathbb{R})$ and consider

$$
\begin{aligned}
& |Q ⿱ Q \iota \iota(r)-Q \mathcal{L}(r)| \leq \int_{0}^{r}|F(r, s, \iota(s))-F(r, s, \kappa(s))| \mathrm{d} s \\
& \leq \int_{0}^{r} f(r, s) \max \left\{|\iota(s)-\kappa(s)|,|\iota(s)-\mathscr{Q} \kappa(s)|,|\kappa(s)-\mathscr{Q} \kappa(s)|, \frac{2|\iota(s)-\mathscr{Q} \kappa(s)| \mid \kappa(s))-\mathscr{Q} \iota(s) \mid}{|\iota(s)-\mathscr{Q} \kappa(s)|+\iota(s)-\kappa(s) \mid}\right\} \mathrm{d} s \\
& \leq \int_{0}^{r} f(r, s) \max \left\{\sup _{s \in[0,1]}|\iota(s)-\kappa(s)|, \sup _{s \in[0,1]}|\iota(s)-Q \mathcal{Q} \kappa(s)|, \sup _{s \in[0,1]}|\kappa(s)-Q \kappa(s)|,\right.
\end{aligned}
$$

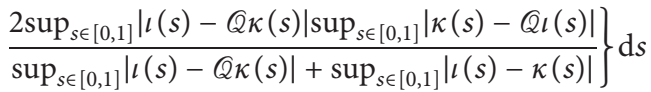

$$
\begin{aligned}
& \leq \max \{d(\iota, \kappa), d(\iota, \mathbb{Q} \kappa), d(\kappa, \mathbb{Q} \kappa),(2 d(\iota, \mathscr{Q} \kappa) d(\kappa, \mathbb{Q} \iota) / d(\iota, Q \mathcal{Q} \kappa)+d(\iota, \kappa))\} \int_{0}^{r} f(r, s) \mathrm{d} s \\
& \leq \lambda \max \{d(\iota, \kappa), d(\iota, \mathbb{Q} \kappa), d(\kappa, \mathbb{Q} \kappa),(2 d(\iota, \mathscr{Q} \kappa) d(\kappa, \mathbb{Q} \iota) / d(\iota, Q \mathcal{Q} \kappa)+d(\iota, \kappa))\} .
\end{aligned}
$$


Consequently,

$$
d(\mathbb{Q} \iota, \mathbb{Q} \kappa) \leq \lambda \max \left\{d(\iota, \kappa), d(\iota, \mathbb{Q} \kappa), d(\kappa, \mathbb{Q} \kappa), \frac{2 d(\iota, \mathbb{Q} \kappa) d(\kappa, \mathbb{Q} \iota)}{d(\iota, \mathbb{Q} \kappa)+d(\iota, \kappa)}\right\}
$$

Using (55), we can write

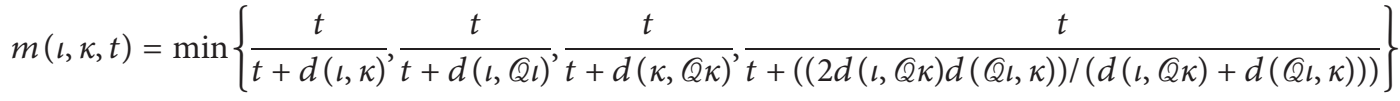

$$
\begin{aligned}
& =\frac{t}{t+\max \{d(\iota, \kappa), d(\iota, \mathbb{Q} \iota), d(\kappa, \mathbb{Q} \kappa),((2 d(\iota, \mathbb{Q} \kappa) d(\mathbb{Q} \iota, \kappa)) /(d(\iota, \mathbb{Q} \kappa)+d(\mathbb{Q} \iota, \kappa)))\}} \\
& \frac{1}{m(\iota, \kappa, t)}-1=\frac{t+\max \{d(\iota, \kappa), d(\iota, \mathbb{Q} \iota), d(\kappa, \mathscr{Q} \kappa),((2 d(\iota, \mathbb{Q} \kappa) d(\mathbb{Q} \iota, \kappa)) /(d(\iota, \mathbb{Q} \kappa)+d(\mathscr{Q} \iota, \kappa)))\}}{t}-1 \\
& =\frac{\max \{d(\iota, \kappa), d(\iota, \mathbb{Q} \iota), d(\kappa, \mathbb{Q} \kappa),((2 d(\iota, \mathbb{Q} \kappa) d(\mathbb{Q} \iota, \kappa)) /(d(\iota, \mathbb{Q} \kappa)+d(\mathbb{Q} \iota, \kappa)))\}}{t} .
\end{aligned}
$$

Therefore,

$$
\begin{aligned}
\frac{1}{\mathscr{F}(\mathbb{Q} \iota, \mathbb{Q} \kappa, t)}-1 & =\frac{d(\mathbb{Q} \iota, \mathbb{Q} \kappa)}{t} \leq \lambda \frac{\max \{d(l, \kappa), d(\iota, \mathbb{Q} \kappa), d(\kappa, \mathbb{Q} \kappa),(2 d(l, \mathbb{Q} \kappa) d(\kappa, \mathbb{Q} \iota) / d(\iota, \mathbb{Q} \kappa)+d(l, \kappa))\}}{t} \\
& =\lambda\left(\frac{1}{m(l, \kappa, t)}-1\right)=\lambda\left(\frac{1}{m(l, \kappa, t)}-1\right) .
\end{aligned}
$$

So, (5) holds for $\eta(k)=\lambda k$ for all $k>0$ with $\alpha(\iota, \kappa, t)=1$, for all $\iota, \kappa \in C([0, I], \mathbb{R})$. As $C([0, I], \mathbb{R})$ is complete, so all the remaining conditions of Theorem 1 hold. Hence, (52) admits a unique solution.

\section{Data Availability}

No data were used to support this study.

\section{Conflicts of Interest}

The authors declare that there are no conflicts of interest regarding the publication of this manuscript.

\section{Authors' Contributions}

All authors contributed equally to the writing of this paper. All authors read and approved the final manuscript.

\section{References}

[1] L. A. Zadeh, "The international journal of fuzzy logic and intelligent systems," Fuzzy Sets, vol. 8, pp. 103-112, 1965.

[2] I. Kramosil and J. Michalek, "Fuzzy metric and statistical metric spaces," Kybernetica, vol. 11, pp. 336-344, 1975.

[3] A. George and P. Veeramani, "On some results in fuzzy metric spaces,” Fuzzy Sets and Systems, vol. 64, no. 3, pp. 395-399, 1994.

[4] B. Samet, C. Vetro, and P. Vetro, "Fixed point theorems for -contractive type mappings," Nonlinear Analysis: Theory, Methods \& Applications, vol. 75, no. 4, pp. 2154-2165, 2012.

[5] D. Gopal and C. Vetro, "Some new fixed point theorems in fuzzy metric spaces, Iran," Journal of Fuzzy System, vol. 11, pp. 95-107, 2014.

[6] J. Ahmad, H. Aydi, and N. Mlaiki, "Fuzzy fixed points of fuzzy mappings via F-contractions and an application," Journal of Intelligent \& Fuzzy Systems, vol. 37, no. 4, pp. 5487-5493, 2019. 
[7] B. Schweizer and A. Sklar, "Statistical metric spaces," Pacific Journal of Mathematics, vol. 10, no. 1, pp. 313-334, 1960.

[8] M. Grabiec, "Fixed points in fuzzy metric spaces," Fuzzy Sets System, vol. 27, pp. 385-389, 1983.

[9] R. Vasuki and P. Veeramani, "Fixed point theorems and Cauchy sequences in fuzzy metric spaces," Fuzzy Sets and Systems, vol. 135, no. 3, pp. 415-417, 2003.

[10] C. Di Bari and C. Vetro, "Fixed points, attractors and weak fuzzy contractive mappings in a fuzzy metric space," Journal of Fuzzy Mathematics, vol. 13, pp. 973-982, 2005.

[11] J. Rodriguez-Lopez and S. Romaguera, "The Hausdorff fuzzy metric on compact sets," Fuzzy Sets and Systems, vol. 147, pp. 273-283, 2004.

[12] J. Jachymski, "Equivalent conditions for generalized contractions on (ordered) metric spaces," Nonlinear Analysis: Theory, Methods \& Applications, vol. 74, no. 3, pp. 768-774, 2011. 